\title{
CONGRUENCE-FREE RIGHT SIMPLE SEMIGROUPS
}

\author{
P. G. TROTTER
}

(Received 5 July, 1976)

Communicated by T. E. Hall

\begin{abstract}
Congruence-free right simple semigroups with idempotents are simple groups or have order 2. It is shown in this paper that there exist congruence-free simple semigroups without idempotents. Some properties of congruences on right simple semigroups, expressed mainly in terms of their unitary and left unitary subsemigroups, are determined. These are used to provide characterizations of congruence-free right simple semigroups.
\end{abstract}

\section{Introduction}

A congruence $\rho$ on $S$ is called proper if and only if $\rho$ is not the identity congruence $\iota$ on $S$ and $\rho$ is not the universal congruence on $S$. $S$ is congruence-free if and only if it has no proper congruences. Tamura (1956) proved that a completely simple or completely 0 -simple semigroup is congruence-free if and only if it is a simple group, a particular regular Rees matrix semigroup, or has order $\leqq 2$. Munn (1974) and Trotter (1974) characterized congruence-free inverse semigroups in terms of idempotent properties; the results have been compared and improved by Munn (1975). Congruence-free regular semigroups with zero have similarly been characterized by Trotter (1976). In this paper we provide some characterizations of congruence-free right simple semigroups as right simple semigroups whose left unitary or unitary subsemigroups satisfy certain restrictions.

Since a right simple semigroup $S$ with idempotents is a right group it can be easily shown that $S$ is congruence-free if and only if $S$ is a simple group or the right zero semigroup of order 2 . We will see that any Baer-Levi semigroup of type $(p, p)$ has a least non-trivial homomorphic image and that this image is a congruence-free right simple semigroup without idempotents.

Congruences on a right simple semigroup $S$ are investigated in section 3 . The results obtained depend mainly on properties of the unitary subsemi- 
groups of $S$. These results are adapted in section 4 to provide the characterizations of congruence-free right simple semigroups.

The terminology, unless otherwise explained, is from Clifford and Preston (1961 and 1967). We use the convention that descriptive words associated with a congruence $\rho$ on $S$ describe the semigroup $S / \rho$. For example if $S / \rho$ is right zero semigroup we say that $\rho$ is a right zero congruence on $S$.

\section{Preliminary results and existence theorem}

Some elementary properties of congruence-free right simple semigroups are derived in this section. It is then shown that congruence-free right simple semigroups without idempotents exist.

LeMma 2.1. Let $S$ be either a right 0 -simple, or right zero, or null semigroup. Then $S$ is congruence-free if and only if $|S| \leqq 2$.

Proof. Suppose $|S|>2$. If $S$ is right 0 -simple then by 2.27 of Clifford and Preston (1961) $S$ has a congruence with classes $S \backslash 0$ and $\{0\}$. If $S$ is right zero and $x, y \in S$ then there is a congruence on $S$ with $\{x, y\}$ for a class. If $S$ is null and $x \in S$ then $\{0, x\}$ is an ideal of $S$. Hence $S$ is congruence-free only if $|S| \leqq 2$. The converse is obvious.

A semigroup $S$ is left reductive if, for $a, b \in S$, whenever $x a=x b$ for all $x \in S$ then $a=b$.

LEMMA 2.2. Any congruence-free semigroup $S$ with $|S|>2$ is left and right reductive.

Proof. Let $\rho$ be the congruence

$$
\rho=\{(y, z) \in S \times S ; y x=z x \text { for all } x \in S\} .
$$

Assume $\rho=S \times S$. Since $S x^{2} \subseteq S x=\left\{x^{2}\right\}, S x^{2}=\left\{x^{2}\right\}$ for all $x \in S$, so $S$ has an ideal of right zeros. Since $S$ is congruence-free then $S$ is a right zero or null semigroup. This contradicts Lemma 2.1 so $\rho=\iota$ and $S$ is right reductive. Dually $S$ is left reductive.

THEOREM 2.3. Let $S$ be a congruence-free right simple semigroup. Then

(i) $S$ has an idempotent if and only if $S$ is a simple group or $|S| \leqq 2$, and

(ii) if $|S|>2$ then $S$ is right cancellative.

Proof. Since a right simple semigroup with an idempotent is a right group then (i) follows from Lemma 2.1. Suppose $|S|>2$ and $a x=b x$ for some $a, b, x \in S$. Since $x S=S$ then $a y=b y$ for all $y \in S$. Hence by Lemma 2.2, $a=b$. 
It will now be shown that there exist congruence-free right simple semigroups without idempotents. Let $X$ be a set of infinite cardinality. Let $B_{X}$ denote the Baer-Levi semigroup of type $(|X|,|X|)$ on $X$. Then $B_{X}$ is the subsemigroup of the full transformation semigroup on $X$, consisting of all one to one transformations $\alpha$ so that $|X| X \alpha|=| X \mid$.

For a subset $Y \subseteq X$ so that $|Y|=|X|$ and for $\alpha, \beta \in B_{X}$ define

$$
d_{Y}(\alpha, \beta)=|(Y \alpha \cup Y \beta) \backslash(Y \alpha \cap Y \beta)| \text {. }
$$

It can be readily checked that $d_{Y}(\alpha, \gamma) \leqq d_{Y}(a, \beta)+d_{Y}(\beta, \gamma)$ for any $\alpha, \beta, \gamma \in B_{X}$. For $\delta \in B_{X}$ let $Z=Y \delta$. Then $Z \subseteq X,|Z|=|X|$ and $d_{Y}(\delta \alpha, \delta \beta)=d_{Z}(\alpha, \beta)$. Also, since $\delta$ is one to one, $d_{Y}(\alpha \delta, \beta \delta)=d_{Y}(\alpha, \beta)$. We can therefore define a congruence $\pi_{X}$ on $B_{X}$ by

$\pi_{X}=\left\{(\alpha, \beta) \in B_{X} \times B_{X} ; d_{Y}(\alpha, \beta)<|X|\right.$ for each $Y \subseteq X$ where $\left.|Y|=|X|\right\}$.

THEOREM 2.4. Let $X$ be a set of infinite cardinality. Then $B_{X} / \pi_{X}$ is a congruence-free right simple semigroup without idempotents.

Proof. For any $\alpha, \beta \in B_{X}$ let $\tau_{\alpha, \beta}$ denote the least congruence on $B_{X}$ so that $(\alpha, \beta) \in \tau_{\alpha, \beta}$. Assume that $(\alpha, \beta) \notin \pi_{X}$. We will see that $\pi_{X}$ is the greatest non-universal congruence on $B_{X}$ by proving that $\tau_{\alpha, \beta}$ is the universal congruence on $B_{X}$. There exists $Y \subseteq X,|Y|=|X|$ so that $d_{Y}(\alpha, \beta)=|X|$. Let $Z=Y \alpha \backslash(Y \alpha \cap Y \beta)$ and assume $|Z|=|X|$; otherwise let $Z=$ $Y \beta \backslash(Y \alpha \cap Y \beta)$. Choose $\zeta \in B_{X}$ so that $X \zeta \subseteq Z \alpha^{-1}$. We have

$$
X \zeta \alpha \cap X \zeta \beta \subseteq Z \cap Z \alpha^{-1} \beta \subseteq Z \cap Y \beta=\square
$$

so the ranges of the transformations $\zeta \alpha$ and $\zeta \beta$ respectively are disjoint. Since $B_{X}$ is right simple there exists $\eta \in B_{X}$ so that $\alpha=\zeta \alpha \eta$. Because $\eta$ is one to one and $X \zeta \alpha \cap X \zeta \beta=\square$ then $X \alpha \cap X \zeta \beta \eta=\square$. So $\alpha$ and $\zeta \beta \eta$ have disjoint ranges. We can now show for any $\gamma \in B_{X}$ that $(\alpha, \gamma) \in \tau_{\alpha, \beta}$. There are two possibilities. Suppose $|X \backslash(X \alpha \cup X \gamma)|=|X|$. Since $\alpha$ and $\zeta \beta \eta$ have disjoint ranges, we choose $\mu \in B_{X}$ so that $X \alpha \mu=X \alpha$ and $X \zeta \beta \eta \mu \subseteq X \backslash(X \alpha \cup X \gamma)$. Then $\zeta \beta \eta \mu$ and $\gamma$ have disjoint ranges. Hence we can choose $\nu \in B_{X}$ so that $\alpha \nu=\zeta \beta \eta \mu$ and $\zeta \beta \eta \nu=. \gamma$. Then

$$
\alpha=\zeta \alpha \eta \mu . \quad \zeta \beta \eta \mu=\zeta \alpha \eta \nu . \quad \zeta \beta \eta \nu=\gamma
$$

and since $(\alpha, \beta) \in \tau_{\alpha, \beta}$ then $(\alpha, \gamma) \in \tau_{\alpha, \beta}$. Alternatively if $|X \backslash(X \alpha \cup X \gamma)|<$ $X$ we can choose $\mu \in B_{X}$ so that $\alpha \mu=\alpha$ while $X \zeta \beta \eta \mu \subseteq X \gamma$. Since $X|X \gamma|=|X|$ we can now choose $\nu, \lambda \in B_{X}$ so that $\alpha \nu=\zeta \beta \eta \mu$ while $\zeta \beta \eta \nu$ and $\gamma$ have disjoint ranges, and then so that $\alpha \lambda=\zeta \beta \eta \nu$ and $\zeta \beta \eta \lambda=\gamma$. Hence

$$
\alpha=\zeta \alpha \eta \mu, \quad \zeta \beta \eta \mu=\zeta \alpha \eta \nu, \quad \zeta \beta \eta \nu=\zeta \alpha \eta \lambda, \quad \zeta \beta \eta \lambda=\gamma
$$


so $(\alpha, \gamma) \in \tau_{\alpha, \beta}$. Thus $\tau_{\alpha, \beta}$ is the universal congruence on $B_{X}$, so $\pi_{X}$ is the greatest non-universal congruence. Hence $B_{X} / \pi_{X}$ is right simple and congruence-free. Mielke (1972) has shown that $B_{X}$ has no non-universal regular congruences. Therefore $B_{X} / \pi_{X}$ is not a simple group. Furthermore, since every semigroup of order 2 is either non-right simple or is regular then $\left|B_{X} / \pi_{X}\right| \neq 2$. Hence by Theorem 2.3 (i), $B_{X} / \pi_{X}$ has no idempotents.

\section{Congruences on right simple semigroups}

In this section we investigate in particular right cancellative left reductive congruences and regular congruences of a right simple semigroup $S$. Such congruences will be seen to be determined by either unitary or certain left unitary subsemigroups of $S$. Recall that a subsemigroup $U$ of $S$ is left unitary if for $x \in S$ and $a, a x \in U$ then $x \in U$. $U$ is unitary if it is also right unitary.

Let $S$ be a right simple semigroup and $\rho$ be a congruence on $S$. For $a \in S$ define

$$
\begin{aligned}
& U_{a, \rho}=\{x \in S ;(a x) \rho=a \rho\} . \\
& V_{a, \rho}=\{x \in S ;(a x b) \rho=(a b) \rho \text { for all } b \in S\} .
\end{aligned}
$$

Clearly $V_{a, \rho} \supseteq U_{a, \rho} \neq \square$ and $\rho$ saturates $V_{a, \rho}$ and $U_{a, \rho}$ (that is, they are both unions of $\rho$-classes). It can be easily checked that $U_{a, \rho}$ and $V_{a, \rho}$ are left unitary subsemigroups of $S$. If $y, x y \in V_{a, \rho}$ for $x \in S$ then $(a(y b)) \rho=(a b) \rho=$ $(a x(y b)) \rho$ for all $b \in S$. Then $x \in V_{a, \rho}$ since $S$ is right simple so $V_{a, \rho}$ is unitary.

For $S \supseteq W \neq \square$ let

$$
\begin{gathered}
\mathscr{P}_{W}=\left\{(a, b) \in S \times S ; x a y \in W \text { if and only if } x b y \in W \text { for } x, y \in S^{\prime}\right\}, \\
\mathscr{R}_{W}=\left\{(a, b) \in S \times S ; a y \in W \text { if and only if } b y \in W \text { for } y \in S^{\prime}\right\} .
\end{gathered}
$$

These are respectively the greatest congruence and greatest right congruence on $S$ that saturate $W$ (see Teissier (1951)).

LEMMA 3.1. Let $S$ be a right simple semigroup. If $V$ is a unitary subsemigroup of $S$ then $\mathscr{P}_{V}$ is a right cancellative left reductive congruence on $S$.

Conversely, let $\rho$ be a right cancellative left reductive congruence on $S$ and let $V=U_{a . \rho}$ for some $a \in S$. Then $V$ is a unitary subsemigroup of $S$ and $\rho=\mathscr{P}_{v}=\mathscr{P}_{x \rho}=\mathscr{R}_{x \rho}$ for any $x \in S$. Furthermore a $=a V$ and $(a x) \rho=$ $a\left(x \mathscr{R}_{V}\right)$.

Proof. Let $V$ be a unitary subsemigroup of $S$ and suppose ( $x a, y a) \in \mathscr{P}_{V}$ for some $x, y, a \in S$. Then $p x a q \in V$ if and only if pyaq $\in V$ for $p, q \in S^{1}$. In particular, since $S$ is right simple then for any $p \in S^{1}$ there is a $t \in S^{1}$ so that pxat, pyat $\in V$. Since $V$ is unitary, if $p x \in V$ then at $\in V$ so $p y \in V$, and 
conversely. We therefore have, since $a S=S$, that pxr $\in V$ if and only if pyr $\in V$ for any $p, r \in S^{1}$, so $(x, y) \in \mathscr{P}_{V}$ and $\mathscr{P}_{V}$ is right cancellative. If $(p u, p v) \in \mathscr{P}_{V}$ for all $p \in S$ and some $u, v \in S$ then $p u s \in V$ if and only if pvs $\in V$ for any $s \in S^{1}$. Furthermore $u r \in V$ if and only if $v r \in V$ for any $r \in S^{1}$ since $u r \in V$ if and only if pur $\in V$ for any $p \in V$. Hence $(u, v) \in \mathscr{P}_{V}$ and $\mathscr{P}_{V}$ is left reductive.

Conversely let $V=U_{a, \rho}$. Since $\rho$ is right cancellative it can be easily checked that $V$ is a unitary subsemigroup of $S$. Since $\rho$ saturates $V$ then $\rho \subseteq \mathscr{P}_{v}$. Suppose $(u, v) \in \mathscr{P}_{v} . S$ is right simple so for $p \in S^{1}$ there is a $q \in S^{1}$ so that $p u q, p v q \in V$. Hence $(a p u q) \rho=a \rho=(a p v q) \rho$. Cancelling, $(a p u) \rho=$ (apv) $\rho$ for all $p \in S^{1} . S / \rho$ is right simple and left reductive so $(u, v) \in \rho$. Therefore $\rho=\mathscr{P}_{V}$. From the definition of $U_{a, p}, a V=a \rho$. Suppose that $(y, z) \in \mathscr{R}_{x \rho}$ for some $x, y, z \in S$. Then there exists $r \in S^{1}$ so that $y r, z r \in x \rho . \rho$ is right cancellative so $(y, z) \in \rho$. Hence $\mathscr{R}_{x \rho} \subseteq \rho \subseteq \mathscr{P}_{x \rho} \subseteq \mathscr{R}_{x \rho}$ so $\rho=\mathscr{P}_{x \rho}=$ $\mathscr{R}_{x p .}$. Suppose $(x, y) \in \mathscr{R}_{V}$ then $x d, y d \in V$ for some $d \in S^{1}$ and axd, ayd $\in$ a $\rho$. Cancelling, $(a x, a y) \in \rho$ so $a\left(x \mathscr{R}_{v}\right) \subseteq(a x) \rho$. Also if $(a x) \rho=(a u) \rho$ then for any $b \in S^{1},(a x b) \rho=a \rho$ if and only if $(a u b) \rho=a \rho$. So $x b \in V$ if and only if $u b \in V$. Hence $(a x) \rho \subseteq a\left(x \mathscr{R}_{v}\right)$.

LEMMA 3.2. Let $S$ be a right simple semigroup and let $\rho$ be a nonuniversal, non right zero congruence on $S$. Then there is a non-universal right cancellative and left reductive congruence $\rho^{*}$ on $S$ so that $\rho \subseteq \rho^{*}$.

Proof. Choose $a \in S$. If $V_{a, \rho}=S$ then $\left(a S U_{a, \rho}\right) \rho=a \rho$ and since $S a \subseteq$ $a S=S$ then $(S \rho)(a \rho)=\left(S a U_{a, \rho}\right) \rho \subseteq\left(a S U_{a, \rho}\right) \rho=a \rho$ so $(S \rho)(a \rho)=a \rho$. But then $S / \rho$ is right simple with a right zero so $S / \rho$ is a right zero semigroup, a contradiction to assumption. Hence $V_{a, \rho} \neq S$. We have seen that $V_{a, \rho}$ is unitary and is saturated by $\rho$. Hence by Lemma 3.1 for $V=V_{a, \rho}, \mathscr{P}_{V}$ is right cancellative and left reductive. Since $\mathscr{P}_{V}$ is the greatest congruence saturating $V$, we may choose $\rho^{*}=\mathscr{P}_{V}$.

A subset $K$ of a semigroup $S$ is a normal complex of $S$ if it is a class of some congruence on $S$. So $K$ is a normal complex if and only if $K$ is a $\mathscr{P}_{K}$-class. For $a \in S$ we define an a-unitary subsemigroup $V$ of $S$ to be a unitary subsemigroup so that $a x \in a V$ for $x \in S$ if and only if $x \in V$, and so that $a \in a V$.

THEOREM 3.3. Let $S$ be a right simple semigroup without idempotents and with no proper group congruences. Then $S$ has a maximal non-regular congruence.

Proof. Choose $a \in S$ and let $\rho$ be a non-regular congruence on $S$. Then $\rho$ is non-universal and by Lemma 3.2 there is a non-universal right cancella- 
tive left reductive congruence $\rho^{*}$ on $S$ so that $\rho \subseteq \rho^{*}$. By Lemma $3.1 \rho^{*}=\mathscr{P}_{v}$ where $V=U_{a . \rho}$ and $a \rho^{*}=a V$. $V$ is $a$-unitary by the definition of $U_{a . \rho}$. Note that $\rho^{*}$ is not a right zero congruence since it is right cancellative. Since $S$ has no non-universal group congruences then by Theorem 1.27 of Clifford and Preston (1961), $S / \rho^{*}$ has no idempotents. So $a \notin V$.

We will now see that $S$ has a maximal $a$-unitary subsemigroup $M$ so that $a \notin M$ and $a M$ is a normal complex. For some index set $I$ let $\left\{U_{i} ; i \in I\right\}$ be a family of $a$-unitary subsemigroups of $S$ so that $a \notin U_{i}$ and $a U_{i}$ is a normal complex of $S$ for each $i \in I$. Assume that the elements of this family form an ascending chain under inclusion and let $U=\bigcup_{i \in I} U_{i}$. It can be readily checked that $U$ is an $a$-unitary subsemigroup of $S, a \notin U$, and $a U$ is a normal complex. For example suppose that $b, c, x b y \in a U$ for some $x, y \in S^{1}$. Then for some $i \in I$ we have $b, c, x b y \in a U_{i}$ and since $a U_{i}$ is a normal complex then $x c y \in a U_{i}$. Hence $(b, c) \in \mathscr{P}_{a U}$ for all $b, c \in a U$, so $a U$ is a $\mathscr{P}_{a U}$-class. Therefore $U$ is an upper bound of the chain and by Zorn's Lemma there is a maximal $a$-unitary subsemigroup $M$ of $S$ so that $a \notin M$ and $a M$ is a normal complex of $S$.

To complete the proof we show that $\mathscr{P}_{a M}$ is a maximal non-regular congruence on $S$. Note that if $\mathscr{P}_{a M}$ is a right zero congruence then the class $a M$ is a subsemigroup of $S$. Then $a M a M \subseteq a M$. But since $M$ is $a$-unitary then $M a M \subseteq M$ and therefore $a \in M$ which is a contradiction. Since, by Theorem 1.27 of Clifford and Preston (1961), the only regular congruences on $S$ are right zero congruences then $\mathscr{P}_{a M}$ is a non-regular congruence on $S$. Suppose that $\sigma$ is a non-regular congruence on $S$ and that $\sigma \supseteq \mathscr{P}_{a M}$. Then as in the first part of the proof there is an a-unitary subsemigroup $W$ of $S$ so that $\mathscr{P}_{w}$ is a non-regular congruence, $\mathscr{P}_{W} \supseteq \sigma \supseteq \mathscr{P}_{a M}, a \mathscr{P}_{W}=a W$ and $a \notin W$. Then $a W \supseteq$ $a M$. Since $W$ is $a$-unitary then $W \supseteq M$. Since $M$ is maximal then $W=M$ and $a W=a M . \mathscr{P}_{a M}$ is the greatest congruence saturating $a M$ so $\mathscr{P}_{W}=\sigma=\mathscr{P}_{a M}$.

We now consider some properties of regular congruences on a right simple semigroup $S$. Since a regular homomorphic image of $S$ is a right group then clearly $S$ has no non-universal regular congruences if and only if $S$ has no non-universal group or right zero congruences. We note that the least cancellative congruence on $S$ is the least group congruence on $S$. Likewise the least left cancellative congruence on $S$ is the least regular congruence on $S$; clearly $S$ has a least right zero congruence.

Teissier (1953) proved that a congruence $\rho$ on a right simple semigroup $S$ is regular if and only if $\rho$ has a class $U$ that is a left unitary subsemigroup of $S$ and so that $U a U \subseteq a U$ for all $a \in S$. It can easily be proved that $\rho$ is a group congruence if and only if $U$ is unitary. Note that $U$ is reflexive if for $x, y \in S$ 
and $x y \in U$ then $y x \in U$. With Clifford and Preston (1967) we have

THEOREM 3.4. Let $S$ be a right simple semigroup. The following are equivalent:

(i) S has no non-universal group congruences.

(ii) The least cancellative congruence on $S$ is universal.

(iii) $S$ has no proper unitary reflexive subsemigroups.

THEOREM 3.5. Let $S$ be a right simple semigroup. The following are equivalent:

(i) S has no non-universal right zero congruences.

(ii) $S$ is not a disjoint union of proper left ideals.

(iii) $S$ has no proper left unitary left ideals.

Proof. Any class of a right zero congruence on $S$ is a left unitary left ideal of $S$. So (iii) implies (i). Conversely assume (i) and suppose $U$ is a left unitary left ideal of $S$. Since $S U=U$ then $a U \subseteq U$ for all $a \in S$. Since $S$ is right simple and $U$ is left unitary then $a U S \supseteq U$ and $a U x \cap U \neq \square$ only if $x \in U$, so $a U=U$ for all $a \in S$. Thus $U a U \subseteq U=a U$ and by the comment preceding Theorem 3.4, $U$ is a class of a regular congruence $\sigma$ on $S$. By (i) $\rho$ is a group congruence. But since $U$ is a left ideal then $U=S$, and we have (i) implies (iii). The equivalence of (i) and (ii) is easily checked.

\section{Characterizations}

We can now derive some characterizations of congruence-free right simple semigroups.

Note that any element $b$ of a right simple right cancellative semigroup $S$ is disjunctive; that is, $\mathscr{P}_{\{b\}}=\iota$. For if $(x, y) \in \mathscr{P}_{\{b\}}$ then there is an $r \in S$ so that $x r=b=y r$, so $x=y$.

Further note that if $e \in V \subseteq S$, since $\mathscr{P}_{V}$ saturates $V$ then $e \mathscr{P}_{V} \subseteq V$.

THEOREM 4.1. Let $S$ be a right simple semigroup with $|S|>2$. Choose $a \in S$ and $e \in U_{a .1}$. Then the conditions $(A),(B)$ and $(C)$ that follow are equivalent.

(A) $S$ is congruence-free.

(B) (i) $S$ is right cancellative and left reductive, and

(ii) for each left unitary subsemigroup $U$ so that $U_{a, \iota} \subset U \subset S$ then $e \mathscr{P}_{U}=\{e\}$.

(C) (i) $S$ is right cancellative and left reductive,

(ii) $S$ has no proper left unitary left ideals, and

(iii) for each unitary subsemigroup $V$ so that $U_{a . \iota} \subset V \subset S$ then $\mathscr{P P}_{V}=\{e\}$. 
Proof. Suppose $S$ satisfies (A). Then clearly $S$ satisfies (B)(ii) and (C) (iii). (B) (i), (C) (i) and (C) (ii) follow from Lemma 2.2 and Theorems 2.3, 3.5 .

Suppose $S$ satisfies (B) and $\rho$ is a non-universal congruence on $S$. Let $U=U_{a, \rho}$. Since $\rho$ saturates $U$ then $\rho \subseteq \mathscr{P}_{L}$. If $U=U_{a, t}$ then by Lemma 3.1 $\iota=\mathscr{P}_{U}=\rho \cdot \rho \neq \mathrm{S} \times \mathrm{S}$ so $U \neq S$. Hence assume $U_{a, \iota} \subset U \subset S$. Then by (B) (i) $e$ is disjunctive and by (B) (ii), $\mathscr{P}_{L}$ saturates $\{e\}$ so $\rho \subseteq \mathscr{P}_{L} \subseteq \mathscr{P}_{\{e\}}=\iota$. Hence $S$ satisfies (A).

Assume (C) and let $\rho$ be a non-universal congruence on $S$. Let $V=V_{a, \rho}$. As in the proof of Lemma 3.2, since $\rho$ is not a right zero congruence then $V \neq S$. Continuing as in the last case we get $\rho \subseteq \mathscr{P}_{V} \subseteq \mathscr{P}_{\{\ell \mid}=\iota$. So $S$ satisfies (A).

Using results from section 3 , the restrictions on the unitary subsemigroups in this theorem can be refined.

THEOREM 4.2. Let $S$ be a right simple semigroup with $a \in S$ and $|S|>2$. Then the conditions (A), (B) and (C) that follow are equivalent.

(A) $S$ is congruence-free.

(B) (i) $S$ is right cancellative,

(ii) $S$ has no proper left unitary left ideal, and

(iii) if $V$ is a proper a-unitary subsemigroup of $S$ and $a V \neq\{a\}$ then $a V$ is not a normal complex of $S$.

(C) (i) $S$ has no proper unitary reflexive subsemigroup of order $>1$,

(ii) $S$ has no proper left unitary left ideal, and

(iii) if $W$ is a maximal a-unitary subsemigroup of $S$ with $a \notin W$ then $\mathscr{P}_{w}=\iota$.

Proof. That (A) implies (B) and (C) is clear from the previous results.

Assume (B), and that $\rho$ is a non-universal congruence on $S$. By (B) (ii), Theorem 3.5 and Lemma 3.2 there is non-universal right cancellative left reductive congruence $\rho^{*} \supseteq \rho$ on $S$. By Lemma $3.1 a \rho^{*}=a V$ where $V=U_{a . \rho}$. $V$ is a proper $a$-unitary subsemigroup of $S$ so by (B) (iii) $a V=\{a\}$. Since $a$ is disjunctive in $S$ then $\rho \subseteq \rho^{*} \subseteq \mathscr{P}_{\{a\}}=\iota$ and (A) follows.

Note that if $V$ and $W$ are unitary subsemigroups of $S$ so that $V \subseteq W$ then $\mathscr{P}_{V} \subseteq \mathscr{P}_{W}$. To see this suppose $(x, y) \in \mathscr{P}_{V}$ and $p x q \in W$ for some $p, q \in S^{1}$. We can choose $r \in S$ so that $p x r \in V$ and then $p y r \in V$. If $q=1$ then $r \in W$ so $p y q \in W$. If $q \neq 1$ then there is an $s \in S$ so that $r s=q$. Then pxrs $\in W$ so $s \in W$ and $p y q=$ pyrs $\in W$.

Assume (C). Note that by (C) (i), if $S$ is a group then $S$ is a simple group (see Clifford and Preston (1967)). Since a regular right simple semigroup is a 
right group then by (C) (i), (C) (ii) and Theorem 3.5, S has no proper regular congruences. In particular if $S$ has a non-universal regular congruence then $S$ is a simple group. Hence assume $S$ is non-regular. By Theorem 3.3 and its proof, $S$ has a maximal non-regular congruence $\mathscr{P}_{M}$ where $M$ is $a$-unitary and $a \notin M$. The Zorn Lemma application of the proof of Theorem 3.3 may be simplified to show that $S$ has a maximal a-unitary subsemigroup $W$ so that $a \notin W$ and $W \supseteq M$. Hence $\mathscr{P}_{M} \subseteq \mathscr{P}_{W}=\iota$. So (C) implies (A).

Note that (A) and (C) are equivalent without the restriction $|S|>2$.

These theorems, in most cases, would be difficult to utilize in testing that a right simple semigroup $S$ is congruence-free. However they do provide a simplification of the criteria of Schein (1966); namely that $S$ is congruencefree if and only if $\mathscr{P}_{K}=\imath$ for each proper subset $K$ of $S$.

\section{References}

A. H. Clifford and G. B. Preston (1961 and 1967), The algebraic theory of semigroups, Vols. I and II, Amer. Math. Soc. Math. Surveys 7.

B. W. Mielke (1972), 'Regular congruences on Croisot-Teissier and Baer-Levi semigroups', $J$. Math. Soc. Japan 24, 539-551.

W. D. Munn (1974), 'Congruence-free inverse semigroups', Quart. J. Math. Oxford (2) 25, 463-484.

W. D. Munn (1975), 'A note on congruence-free inverse semigroups', Quart. J. Math. Oxford (2) 26, 385-387.

B. M. Schein (1966), 'Homomorphisms and subdirect decompositions of semigroups', Pacific $J$. Math. 17, 529-547.

T. Tamura (1956), 'Indecomposable completely simple semigroups except groups', Osaka Math. $J$. $8,35-42$.

M. Teissier (1951), 'Sur les équivalences régulières dans les demi-groupes', C. R. Acad. Sci. Paris 232, 1987-1989.

M. Teissier (1953), 'Sur les demi-groupes ne contenant pas d'élément idempotent', C. R. Acad. Sci. Paris 237, 1375-1377.

P. G. Trotter (1974), 'Congruence-free inverse semigroups', Semigroup Forum 9, 109-116.

P. G. Trotter (1976), 'Congruence-free regular semigroups with zero', Semigroup Forum 12, 1-5.

University of Tasmania,

Hobart, Australia. 